\title{
Dieta de Sciurus ingrami Thomas (Rodentia, Sciuridae) em um remanescente de Floresta com Araucária, Paraná, Brasil ${ }^{1}$
}

\author{
João M. D. Miranda ${ }^{2}$ \\ ${ }^{1}$ Contribuição número 1612 do Departamento de Zoologia, Universidade Federal do Paraná. \\ 2 Laboratório de Biodiversidade, Ecologia e Conservação de Animais Silvestres, Departamento de Zoologia, Universidade \\ Federal do Paraná. Caixa Postal 19020, 81531-980 Curitiba, Paraná, Brasil. E-mail: guaribajoao@yahoo.com.br
}

\begin{abstract}
Diet of Sciurus ingrami Thomas (Rodentia, Sciuridae) on a Araucauria Pine Forest remnant, Paraná, Brazil. This study was conducted between February, 2002 and January, 2003 in a remnant of Araucaria Pine Forest located in the District of Bugre, Municipality of Balsa Nova, Paraná, Brazil. The goal of this study was to survey the species used as food items by Sciurus ingrami Thomas, 1901 throughout a year. Ten $(\mathrm{N}=10)$ species were recorded in the diet of these squirrels, belonging to seven plant families. Squirrels preyed on seeds of three plant species and fed on three exotic species, indicating good adaptability to disturbed habitats. KEY WORDS. neotropical squirrel, olive-colored squirrel, mammal ecology, seed predation.
\end{abstract}

RESUMO. Este estudo foi realizado entre fevereiro de 2002 e janeiro de 2003, em um remanescente de Floresta com Araucária, localizado no Distrito do Bugre, Município de Balsa Nova, Paraná, Brasil. O objetivo foi conhecer as espécies utilizadas na alimentação por Sciurus ingrami Thomas, 1901 no decorrer de um ano. Foram registradas dez (N $=10$ ) espécies na dieta dos serelepes, pertencentes a sete famílias botânicas. Os serelepes predaram sementes de três espécies e se alimentaram de três espécies exóticas, mostrando adaptabilidade a ambientes alterados.

PALAVRAS CHAVE. serelepe, caxinguelê, ecologia de mamíferos, predação de sementes.

O serelepe, Sciurus ingrami Thomas, 1901 tem sua distribuição desde o sul do estado da Bahia até o Rio Grande do Sul (OliveIrA Pinto 1931, Moojen 1952, Cabrera 1961, Lange \& Jablonski 1981). Assim como para S. ingrami (Bordignon \& MonTeIRo-Filho 1997), pouco se conhece a respeito das outras espécies dos esquilos neotropicais (Gurnell 1987, Emmons \& FeER 1997). A ecologia e o comportamento desta espécie em vida livre são pouco conhecidos (Paschoal \& Galetti 1995, Bordignon \& Monteiro-Filho 1999, 2000a, b, Alvarenga \& Talamoni 2005, Alvarenga \& Talamoni no prelo). Sendo que parte do conhecimento sobre esta espécie se baseia em dados de cativeiro (Bordignon et al. 1996), assim, o objetivo deste trabalho foi estudar a dieta de Sciurus ingrami em vida livre, colaborando com maiores informações sobre a espécie.

\section{MATERIAL E MÉTODOS}

O presente estudo foi realizado em um remanescente de Floresta com Araucária (Floresta Ombrófila Mista) com aproximadamente 700ha, localizado no Distrito do Bugre, Município de Balsa Nova, Estado do Paraná, Sul do Brasil (25²9'52"W e 49 39'24"S). O clima na região é Cfb segundo a classificação de Köpen (IAPAR 1978). A temperatura média anual de $17^{\circ} \mathrm{C}$ oscila entre a média de $14^{\circ} \mathrm{C}$ no inverno e $21^{\circ} \mathrm{C}$ no verão, a precipitação anual média de $1600 \mathrm{~mm}$ (dados obtidos na Estação
Meteorológica do Município da Lapa, aproximadamente $35 \mathrm{Km}$ da área de estudo). A natureza topográfica da área é de superfície bastante acidentada, com inclinações de até $90^{\circ}$, associada à presença de pequenos cânions. A maior altitude da área é de $1145 \mathrm{~m}$ enquanto que a menor é de $935 \mathrm{~m}$, mostrando um desnível de mais de $100 \mathrm{~m}$.

A área de estudo está inserida na Área de Proteção Ambiental (APA) Estadual da Escarpa Devoniana, sendo que esta escarpa é o limite entre o primeiro e o segundo planaltos paranaenses (MAACK 1968). A região faz parte da bacia do Alto Rio Iguaçu, apresentando alguns riachos afluentes da margem direita do Rio Iguaçu (MAAcK 1968). Grande parte da região foi desmatada até o fim da década de 1970, por conta da utilização da madeira da floresta para aquecer os fornos de cerâmica do Município de Campo Largo (capital paranaense da cerâmica). Assim, parte da cobertura florestal hoje presente é uma mata em regeneração, associada a porções menos impactadas principalmente em locais onde a própria inclinação da Escarpa Devoniana tornou inacessível o desmate.

Entre fevereiro de 2002 e janeiro de 2003 foram feitas fases de campo quinzenais com duração média de três dias cada, com o objetivo de observar e acompanhar os serelepes em vida livre e registrar os itens utilizados em sua dieta. Observações 
naturalísticas foram feitas adotando o método de todas as ocorrências (Altmann 1974), registrando em caderneta de campo quais as espécies eram utilizadas na alimentação de Sciurus ingrami. A identificação da espécie foi feita sempre que possível em campo, quando essa identificação não foi possível, as árvores foram marcadas e foram coletados frutos e folhas, os quais foram devidamente herborizados para identificação em laboratório e comparadas a coleções de referência.

\section{RESULTADOS}

Das 24 visitas a campo, em apenas 13 foram observados e acompanhados serelepes, sendo que somente o mês de novembro ficou sem ter registros. Nestas 13 fases foram feitas 25 observações de animais se alimentando em um total de três horas e 48 minutos. Foram registradas dez $(\mathrm{N}=10)$ espécies sendo utilizadas na alimentação de $S$. ingrami, pertencentes a sete $(\mathrm{N}=7)$ famílias botânicas (Tab. I). A dieta do serelepe foi baseada principalmente em frutos, associada também ao consumo de sementes de Araucaria angustifolia (Bertol.) O. Kuntze - araucária, de Syagrus romanzoffiana (Cham.) Glassman - jerivá e de Pinus elliottii Engelm. - pinus. Houve também o consumo de três espécies exóticas, sendo elas: o próprio pinus, Dispyrus kaki Linnaeus - caqui e Eryobotrya japonica (Thumb.) Lindl. ameixa-amarela. As observações indicam que há uma mudança em sua dieta de acordo com a disponibilidade dos itens alimentares, que são todos itens sazonais (Tab. I). A tabela II mostra comparativamente as espécies pertencentes à dieta de $S$. ingrami apresentadas neste e em outros trabalhos.

\section{DISCUSSÃO}

De maneira geral, os serelepes se alimentaram de frutos e de sementes, como já descrito para a maioria das espécies de esquilos (GuRNell 1987). As dez espécies registradas neste estudo para a dieta de $S$. ingrami certamente representam uma lista parcial de espécies utilizadas pelos serelepes no remanescente florestal do Bugre. Isso, não só tendo em vista o pequeno número de horas de observação, mas também porque outros estudos mostram outras espécies, além de outros itens alimentares. Paschoal \& Galetti (1995), por exemplo, registraram 14 espécies no Estado de São Paulo, e Bordignon \& Monteiro-Filho (1999) identificaram dez espécies vegetais (além de fungos, cascas de árvores, flores e itens não identificados) no Estado do Paraná. Das dez espécies vegetais presentes na dieta do serelepe no estudo de Bordignon \& Monteiro-Filho (1999), quatro foram também encontradas no presente trabalho ( $S$. romanzoffiana, A. angustifolia, E. japonica e P. elliottii), provavelmente por se tratar de uma mesma formação vegetacional (Floresta com Araucária). Durante os meses de primavera e de verão a dieta de $S$. ingrami foi mais variada, sendo registrada a presença de oito espécies. Já nos meses de outono e de inverno, sua dieta foi mais restrita, com apenas quatro espécies. Duas espécies de Arecaceae (S. romanzoffiana e G. schottiana) aparecem tanto em um período como em outro e são espécies que apresentam tipicamente mais de um pico anual de frutificação. Destaca-se aqui o consumo das sementes de araucária durante o outono e inverno, que pode ser um fator importante para a passagem do inverno (que nesta região do país é bem rigoroso), pois essa é uma boa fonte de carboidratos e lipídios, ou seja, possui um alto valor energético (JARDIM \& OliveIRa 2000). Esta variação sazonal com um maior número de espécies aparecendo na dieta do serelepe durante o verão também foi vista por BoRDignON \& Monteiro-Filho (1999), o que não foi visto por Paschoal \& GaLetTI (1995), apesar de mostrarem uma variação sazonal nas espécies consumidas por $S$. ingrami.

Apesar de este estudo não ter um caráter quantitativo, a presença dos frutos e sementes de jerivá em vários meses mostra que esta é uma espécie importante na dieta dos serelepes. Isso também foi visto por outros autores em outras localidades (Paschoal \& Galetti 1995, Bordignon \& Monteiro-Filho 1999, Alvarenga \& TALAMONI no prelo), o que corrobora a importância desta palmeira na dieta dos serelepes. Além disso, o jerivá é uma espécie importante não somente para os serelepes mas também para outros mamíferos frugívoros (PAssos 1999), como por exemplo Alouatta guariba (Humboldt, 1812) (Aguiar et al. 2003, Miranda \& Passos 2004), Leontopithecus chrysopygus (Mikan, 1823) (PAssos 1999) e Cerdocyon thous (Linnaeus, 1758) (FACURE \& Monteiro-Filho 1996, Rocha et al. 2004).

Neste estudo além de Syagrus romanzoffiana aparecem também os frutos e sementes de Geonoma shottiana - guaricana (Arecaceae) o que pode mostrar que o consumo de frutos e sementes de Arecaceae na dieta de Sciurus ingrami parece ser uma constante em todas as áreas estudadas (GALETTi et al. 1992, Paschoal \& Galetti 1995, Bordignon \& Monteiro-Filho 1999, Alvarenga \& Talamoni no prelo).

A presença de Geonoma schottiana na dieta de $S$. ingrami pode corroborar a idéia de que os serelepes são especialistas em se alimentar de frutos com endocarpo lignificado (PASCHOAL \& GalEtTr 1995). Assim o consumo de sementes de araucária e de pinus no presente estudo também pode dar suporte a essa idéia, pois são todos alimentos lignificados.

Além de consumirem algumas espécies de sementes, os serelepes também podem atuar como dispersores de sementes (Paschoal \& Galetti 1995, Bordignon \& Monteiro-Filho 1999, 2000b, Silva \& TaBarelli 2001), já que eles possuem o hábito de enterrar algumas sementes, principalmente de jerivá e de araucária, dentre outras (PASCHOAL \& GALETTI 1995, BORDIGNON \& Monteiro-Filho 1999, 2000b).

A utilização das sementes de pinus e de araucária por $S$. ingrami também foi vista por Bordignon \& MonTeiro-FilHo (1999), que mostram (e aqui é corroborado) que a retirada das sementes do cone do pinus pelo serelepe foi similar ao feito por Sciurus aberti Woodhouse, 1853 e $S$. carolinensis Gmelin, 1788 ao consumir estes itens no hemisfério norte (GURNELL 1987).

Oito das dez espécies registradas no presente estudo para a alimentação de $S$. ingrami apareceram também na dieta de 
Tabela I. Espécies utilizadas na alimentação de Sciurus ingrami no período de fevereiro de 2002 a janeiro de 2003, em um remanescente de Floresta com Araucária no sul do Brasil.

\begin{tabular}{|c|c|c|c|}
\hline Espécie & Nome popular & Item alimentar & Mês do registro \\
\hline \multicolumn{4}{|l|}{ Annonaceae } \\
\hline Rollinia sylvatica (A. St. Hill.) Mart. & Araticum & Frutos & Jan \\
\hline \multicolumn{4}{|l|}{ Araucariaceae } \\
\hline Araucaria angustifolia (Bertol.) O. Kuntze & Araucária & Sementes & Mai, jun, jul \\
\hline \multicolumn{4}{|l|}{ Arecaeae } \\
\hline Syagrus romanzoffiana (Cham.) Glassman & Jerivá & Frutos e sementes & Jan, fev, mai, jun, out, dez \\
\hline Geonoma schottiana Mart. & Guaricana & Frutos & Jan, mai \\
\hline \multicolumn{4}{|l|}{ Ebenaceae } \\
\hline Diospyrus kaki Linnaeus F.* & Caqui & Frutos & Abr, mai, jun \\
\hline \multicolumn{4}{|l|}{ Mimosaceae } \\
\hline Inga sessilis (Vell.) Mart. & Ingá-macaco & Sementes & Jan \\
\hline \multicolumn{4}{|l|}{ Pinaceae } \\
\hline Pinus elliottii Engelm.* & Pinus & Sementes & Mar, abr \\
\hline \multicolumn{4}{|l|}{ Rosaceae } \\
\hline Eryobotrya japonica (Thumb.) Lindl.* & Ameixa-amarela & Frutos & Ago, set \\
\hline Prunus sellowii Koehne & Pessegueiro-bravo & Frutos & Jan, dez \\
\hline \multicolumn{4}{|l|}{ Myrtaceae } \\
\hline Psidium cattleianum Sabini & Araçá & Frutos & Fev \\
\hline
\end{tabular}

* Espécies exóticas.

Tabela II. Espécies presentes na dieta de Sciurus ingrami em alguns estudos: (A) PAsCHOAL \& Galetti (1995), (B) BordiGnON \& Monteiro-FilHo (1999), (C) Alvarenca \& Talamoni (no prelo), (D) presente estudo.

\begin{tabular}{|c|c|c|c|c|c|c|}
\hline & Espécie & Nome popular & A & B & $\mathrm{C}$ & $\mathrm{D}$ \\
\hline Annonaceae & Rollinia sylvatica (A. St. Hill.) Mart. & Araticum & & & & $x$ \\
\hline Araucariaceae & Araucaria angustifolia (Bertol.) O. Kuntze & Araucária & & $x$ & & $x$ \\
\hline \multirow[t]{3}{*}{ Arecaceae } & Syagrus romanzoffiana (Cham.) Glassman & Jerivá & $x$ & $x$ & $x$ & $x$ \\
\hline & Geonoma schottiana Mart. & Guaricana & & & & $x$ \\
\hline & Butia eriospatha Becc. & Butiá & & $x$ & & \\
\hline Boraginaceae & Cordia ecalyculata Vell. & Chá-de-bugre & $\mathrm{x}$ & & & \\
\hline Combretaceae & Terminalia cattapa L.* & Chapéu-de-sol & $x$ & & & \\
\hline Ebenaceae & Diospyrus kaki Linaeus F.* & Caqui & & & & $\mathrm{x}$ \\
\hline \multirow[t]{2}{*}{ Euphorbiaceae } & Croton floribundus Spreng. & capixingui & $\mathrm{x}$ & & & \\
\hline & Dalechampia pentaphylla Lam. & - & $\mathrm{x}$ & & & \\
\hline \multirow[t]{2}{*}{ Fagaceae } & Castanea sativa Mill.* & Castanheira & & $x$ & & \\
\hline & Quercus pedunculata Ehrh. * & Carvalho-vermelho & & $x$ & & \\
\hline Lauraceae & Ocotea corimbosa (Meissner) Mez & Canela-fedida & $x$ & & & \\
\hline Lythaceae & Lafoensia pacari St, Hil. & dedaleiro & & & $x$ & \\
\hline Malpighiaceae & Dicella bracteosa (A. Juss.) Griseb. & trepadeira & $x$ & & & \\
\hline Meliaceae & Cabralea canjerana (Vell.) Mart. & canjerana & $x$ & & & \\
\hline Mimosaceae & Inga sessilis (Vell.) Mart. & Ingá-macaco & & & & $x$ \\
\hline Moraceae & Ficus enormis (Miq.) Miq. & figueira & $x$ & & & \\
\hline \multirow[t]{3}{*}{ Myrtaceae } & Psidium cattleianum Sabini & Araçá & & & & $x$ \\
\hline & Eugenia uniflora Linnaeus & Pitanga & & $x$ & & \\
\hline & Eugenia ligustrina (Sw.) Willd. & pitanga & $x$ & & & \\
\hline
\end{tabular}


Tabela II. Continuação.

\begin{tabular}{|c|c|c|c|c|c|c|}
\hline & Espécie & Nome popular & A & B & $\mathrm{C}$ & $\mathrm{D}$ \\
\hline \multirow[t]{2}{*}{ Pinaceae } & Pinus elliottii Engelm.* & Pinus & & & & $\mathrm{x}$ \\
\hline & Pinus taeda Linnaeus* & Pinus & & $x$ & & \\
\hline Polygalaceae & Diclidanthera laurifólia Mart. & - & $x$ & & & \\
\hline Rhamnaceae & Rhamnus sphaerosprema Sw. & Fruto-do-pombo & & $x$ & & \\
\hline \multirow[t]{2}{*}{ Rosaceae } & Eryobotrya japonica (Thumb.) Lindl.* & Ameixa-amarela & & $x$ & & $\mathrm{x}$ \\
\hline & Prunus sellowii Koehne & Pessegueiro-bravo & & & & $x$ \\
\hline Sapotaceae & Chrysophyllum gonocaroum (Mart. \& Eichl.) Engl. & Guatambu-de-leite & $x$ & & & \\
\hline Symplocaceae & Symplocos uniflora (Pohl) Benth. & - & & $x$ & & \\
\hline Ulmaceae & Celtis iguanaea (Jacq.) Sarg. & Unha-de-gato & $x$ & & & \\
\hline Verbenaceae & Citharexyllum myrianthum Cham. & - & $x$ & & & \\
\hline
\end{tabular}

*Espécies exóticas.

Alouatta guariba (Humboldt, 1812) na mesma área de estudo (Miranda \& Passos 2004), porém não foi observado nenhum comportamento agonístico entre essas duas espécies em nenhum dos dois estudos.

A utilização de espécies exóticas (P. elliotii, E. japonica e D. kaki) pelos serelepes, assim como visto em outros estudos (Tab. II) (Paschoal \& Galetti 1995, Bordignon \& Monteiro-Filho 1999, Alvarenga \& Talamoni no prelo), demonstra um caráter oportunista e capacidade adaptativa desta espécie de roedor para adequar-se a ambientes alterados.

\section{AGRADECIMENTOS}

Agradeço aos Profesores Fernando C. Passos e Emygdio L.A. Monteiro-Filho pelo incentivo e pela leitura crítica dos manuscritos. A Itiberê P. Bernardi e Rodrigo F. Moro-Rios pelo auxílio em campo.

\section{REFERÊNCIAS BIBLIOGRÁFICAS}

Aguiar, L. M.; N. R. Reis, G. Ludwig \& V. J. Rocha. 2003. Dieta, área de vida, vocalizações e estimativas populacionais de Alouatta guariba em um remanescente florestal no Norte do estado do Paraná. Neotropical Primates, Washington, 11 (2): 78-86

Altmann, J. 1974. Observational study of behavior: sampling methods. Behaviour, Leiden, 40: 227-267.

Alvarenga, C.A. \& S.A. Talamoni. 2005. Nests of the brazilian squirrel Sciurus ingrami Thomas (Rodentia, Sciuridae). Revista Brasileira de Zoologia, Curitiba, 22 (3): 816-818.

Alvarenga, C.A. \& S.A. Talamoni. (no prelo). Foranging behavior of the Brazilian squirrel Sciurus aestuans Linnaeus, 1766 (Rodentia, Sciuridae). Acta Theriologica, Bialowieza.

Bordignon, M.; T.C.C. Margarido \& R.R. LAnge. 1996. Formas de abertura dos frutos de Syagrus romanzoffiana (Chamisso) Glassman efetuadas por Sciurus ingrami Thomas (Rodentia, Sciuridae). Revista Brasileira de Zoologia, Curitiba, 13 (4):
821-828.

Bordignon, M. \& E.L.A. Monteiro-Filho. 1997. Comportamentos e atividade diária de Sciurus ingrami (Thomas) em cativeiro (Rodentia, Sciuridae). Revista Brasileira de Zoologia, Curitiba, 14 (3): 707-722.

Bordignon, M. \& E.L.A. Monteiro-Filho. 1999. Seasonal food resources of the squirrel Sciurus ingrami (Thomas, 1901) in a secondary aracucaria forest in southern Brazil. Studies on Neotropical Fauna and Environment, Tübingen, 34: 137140.

Bordignon, M. \& E.L.A. Monteiro-Filho. 2000a. Behaviour and daily activity of the squirrel Sciurus ingrami in a secondary araucaria forest in southern Brazil. Journal of Canadian Zoology, Toronto, 78 (10): 1732-1739.

Bordignon, M. \& E.L.A. Monteiro-Filho. 2000b. O serelepe Sciurus ingrami (Sciuridae: Rodentia) como dispersor do pinheiro do Paraná Araucaria angustifolia (Araucariaceae: Pinophyta). Arquivos de Ciências Veterinária e Zoologia UNIPAR, Umuarama, 3 (2): 139-144.

Cabrera, A. 1961. Catalogo de los Mamiferos de América del Sur. Revista del Museo Argentino de Ciencias Naturales Bernardino Rivadavia, Buenos Aires, 4 (2): 309-732.

Emmons, L. H \& F. Feer. 1997. Neotropical rainforest mammals. A field guide. Chicago, The University of Chicago Press, $307 p$.

Facure, K.G. \& E.L.A. Monteiro-Filho. 1996. Feeding habits of the crab-eating fox, Cerdocyon thous (Carnivora, Canidae), in a suburban area of southeastern Brazil. Mammalia, Paris, 60 (1): 147-149.

Galetti, M.; M. Paschoal \& F. Pedroni. 1992. Predation on palm nut (Syagrus romanzoffiana) by squirrels (Sciurus ingrami) in south-east Brazil. Journal of Tropical Ecology, Cambridge, 8: 121-123.

GuRNELL, J. 1987. The natural history of squirrels. Londres, Christopher Helm, 201p.

IAPAR. 1978. Cartas climáticas básicas do estado do Paraná. 
Londrina, Instituto Agronômico do Paraná, 38p.

Jardim, M.M.A. \& L.F.B. Oliveira. 2000. Aspectos ecológicos e do comportamento de Alouatta fusca (Geoffroy, 1812) na Estação Ecológica de Aracurí, RS, Brasil, p. 151-169. In: C. Alonso \& A. Langguth (Eds). A Primatologia no Brasil - 7. João Pessoa, Sociedade Brasileira de Primatologia, Editora Universitária Universidade Federal da Paraíba, 360p.

LANGe, R.B. \& E.F. Jablonski. 1981. Lista prévia dos Mammalia do Estado do Paraná. Estudos de Biologia, Curitiba, 4: 1-35.

MAACK, R. 1968. Geografia física do Estado do Paraná. Curitiba, Max Roesner, 350p.

MiRanda, J.M.D. \& F.C. Passos. 2004. Hábito alimentar de Alouatta guariba (Humboldt) (Primates, Atelidae) em Floresta de Araucária, Paraná, Brasil. Revista Brasileira de Zoologia, Curitiba, 21 (4): 821-826.

Moojen, J. 1952. Os roedores do Brasil. Rio de Janeiro, Instituto Nacional do Livro, 214p.

Oliveira Pinto, O.M. 1931. Ensaio sobre a fauna de Sciurídeos do Brasil. Revista do Museu Paulista, São Paulo, 17 (1): 263-319.

Recebido em 11.III.2005; aceito em 21.XI.2005.
Paschoal, M. \& M. Galetti. 1995. Seasonal food use by Neotropical Squirrel Sciurus ingrami in Southeastern Brazil. Biotropica, Lawrence, 27 (2): 268-273.

PAssos, F.C. 1999. Dieta de um grupo de mico-leão-preto, Leontopithecus chrysopygus (Mikan) (Mammalia, Callitrichidae), na Estação Ecológica dos Caetetus, São Paulo. Revista Brasileira de Zoologia, Curitiba, 16 (Supl. 1): 269-278.

Rocha, V.J.; N.R. Reis \& M.L. Sekiama. 2004. Dieta e dispersão de sementes por Cerdocyon thous (Linnaeus) (Carnivora, Canidae), em um fragmento florestal no Paraná, Brasil. Revista Brasileira de Zoologia, Curitiba, 21 (4): 871-876.

Silva, J.A. \& S.A. Talamoni. 2003. Diet adjustments of maned wolves, Chrysocyon brachyurus (Illiger) (Mammalia, Canidae), subjected to supplemental feeding in a private natural reserve, Southeastern Brazil. Revista Brasileira de Zoologia, Curitiba, 20 (2): 339-345.

Silva, M.G. \& M. TABARelli. 2001. Seed dispersal, plant recruitment and spatial distribution of Bactris acanthcarpa Martius (Arecaceae) in a remnant of Atlantic forest in northeast Brazil. Acta Oecologica, Londres, 22: 259-268. 